\title{
Reply to Quiggin and Robson
}

\section{Warwick McKibbin}

Alex Robson makes a number of important points on the climate change debate: policy needs to be focused on expected costs and expected benefits. I agree completely with this. He claims that no credible economist has demonstrated that there are gains from Australia reducing emissions. The logic of this position is clear in the sense that under perfect certainty Australia is too small to make much difference. However, under uncertainty this statement is misleading because the lack of a clear policy on pricing carbon has meant that critical investment decisions on energy infrastructure have been delayed. This has caused potential economic losses because of the policy uncertainty. Alex could not argue that uncertainty has no economic costs. Otherwise why do insurance markets exist? A clear policy position that enables firms to plan investment and enables risk management - even if climate change does not turn out to be a serious problem - can still have economic benefits. This is why industry has been calling for a clear policy and why they are willing to take on carbon prices (although with a safety valve to control costs). You need to have a clear policy framework with low and controllable costs while you gather more information. This is the approach taken in our research. The point is not whether climate change is true or not - it will be many decades before we can be a little more certain. Given the uncertainty, there needs to be a policy response that lines up expected benefits of taking action with the expected costs. Again the logic that there are no expected benefits of taking action is clearly not true when economic decisions are being distorted by lack of a clear policy framework in Australia. The Task Group approach provides a better framework than we currently have.

The second point as to why an Australian approach to replace the Kyoto strategy is a good idea is that if Australia can design a policy regime that can be implemented in the major emitting countries such as China and India then the gains to Australia are not trivial. This is exactly what the Prime Minister's Task Group approach has the potential to do. It may not eventually be adopted elsewhere but I think the chances are high given the alternatives. If it is not proposed then it will definitely not be adopted.

There is also a problem with the argument that the optimal policy is one in which Australia only adapts. The correct definition of optimal policy is where the marginal cost of mitigation is equal to the marginal cost of adaptation is equal to the expected marginal benefit. ${ }^{1}$ The problem is that benefits of mitigation

\footnotetext{
${ }^{1}$ See McKibbin W. and P. Wilcoxen 2003, 'Climate Policy and Uncertainty: The Role of Adaptation versus Mitigation' in Living With Climate Change: Proceedings of a National Conference on Climate Change Impacts and Adaptation, National Academies Forum, Canberra.
} 
undertaken now are only observed many decades hence and adaptation is undertaken at some time in the future. Under uncertainty there will always be an expected gain from some mitigation now and some adaptation later.

John Quiggin also enters the debate on whether the sceptics are correct. This is not a useful debate to have because whether they are correct or not (we can't know that with any certainty) - the existence of manifest uncertainty implies that some policy action is required. Also it seems odd to continue to argue over whether Australia should ratify the Kyoto Protocol when the Kyoto Protocol is in the process of being evolved into something completely different. It demonstrably has not succeeded (where 'success' is defined as changing global emissions) for reasons that many economists have predicted. It focused only on environmental outcomes without enough design features to take into account the trade-off between economic costs and environmental benefits. Or, to put it another way, it did not adequately line up the expected benefits with the expected costs in a world of enormous uncertainty.

Arguing that Australia's targets under Kyoto were exceptionally generous is misleading. It is not the absolute target that is the measure of generosity, but it is the relative ease of meeting that target. Australia was allowed a rise in emissions relative to 1990 but it was nonetheless a significant cut in emissions relative to what otherwise would be the case in the period 2008 to 2012 . Contrast that to the fact that Europe had the benefits of a collapse in the UK coal industry, together with the collapse of East Germany, to lead to a much lower baseline emissions profile.

Why ratifying Kyoto or not is currently relevant to moving forward with a new global approach to replace a failed Kyoto approach is completely unclear to me. I am sure John would not argue that being part of the war in Iraq was a good policy because we are in the tent deciding what to do next. His discussion about the global climate debate is also several years out of date. No country is seriously considering border taxes against non-complying countries since most countries inside Kyoto are not close to hitting their targets. The world, and the debate on climate change policy, is rapidly moving on from the Kyoto Protocol arguments of a decade ago. 